\title{
Reliable and Efficient Routing Mechanisms for Vehicular ad-hoc Network
}

\author{
Venkatamangarao Nampally ${ }^{1}$ and M. Raghavender Sharma ${ }^{2}$ \\ ${ }^{1} \mathrm{Ph}$. D. Scholar, Department of Computer Science, University College of Science \\ Osmania University, Hyderabad, Telangana, India \\ ${ }^{2}$ Vice Principal and HOD, Department of Statistics, University College of Science, \\ Osmania University, Hyderabad, Telangana, India \\ E-Mail: n.venkat013@gmail.com,drmrsstatou@gmail.com
}

\begin{abstract}
In order to provide stable connections among nodes, a routing protocol is necessary in VANET system. Dynamic topology and frequent disconnection makes difficult to design an efficient routing protocol for VANET to route information among nodes. The survey of routing protocols in VANET system helps in understanding the concepts of smart intelligent transport system (ITS). It is observed that carry-and-forward is the key consideration and main function of an efficient routing protocol in VANET system. This paper explains various routing protocols for VANET system.

Keywords: Routing protocol, Stable, ITS, Topology, Proactive, Reactive, GPSR, GSR
\end{abstract}

\section{INTRODUCTION}

In order to improve performance of network and decrease power consumption in VANET system, routing protocol need to be defined. Routing is process of sending Information from sender to receiver i.e., it is the process of exchanging information between source and destination. The foremost objective of routing protocols is to attain short communication time while using the least amount of network resources [1]. The concept of routing is involved in two functions: first is determining optimal paths, second is transferring the information in the form of packets. Analysis of traditional routing protocols for MANETs demonstrated that their performance is poor in VANETs. Due to the highly dynamic nature of the nodes, efficient routing is a key challenge in VANET system. So, there is need for a routing protocol which provides better information delivery without route breakage. The main problem with the protocols is their route instability.

The traditional view of routing protocols is node-centric view. It means that an established route is a fixed succession of nodes between source and destination. An effective routing protocol directly affects the efficiency of packet transmission. Packets are dropped and the overhead increases which leads to low delivery ratio and high transmission delay [2]. The data being gathered and exchanged by the vehicles requires some protocols or rules through which transmission can take place in a systematic and organized way. The data exchange between nodes in a VANET happens via routing protocols. These protocols define how a packet of data will be distributed among different nodes. The ultimate goal of routing protocols is to select optimal path with minimum cost [3]. Several factors make routing more challenge in VANET.

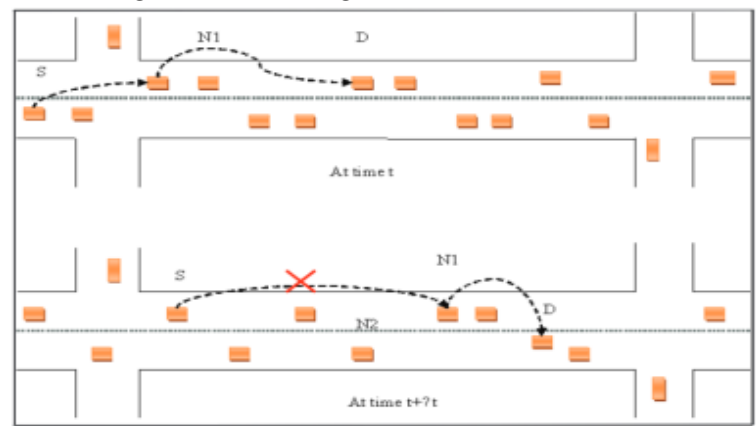

Fig. 1 Pre-established Routes in VANET System

\section{A. Routing Process}

The routing process defines how to use routing metrics to calculate routes. In addition, it defines how often routes must be recalculated or updated, to adapt to network conditions. Moreover, it controls the initiation of routing metrics measurement process. Various routing metrics can be used as indicators for traffic and network conditions. However, the performance of traffic aware routing depends mainly on the utilized routing metrics and the way of measuring these metrics. In particular, accurate measuring of routing metrics will give more realistic indications about traffic conditions and result in more efficient routing. In addition, providing routing metrics on time and with least network overhead, whenever they are required for routing, is critical for efficient routing [4].

\section{B. Forwarding Mechanism}

In all routing protocols, packets need to be forwarded to next-hop or next junction based on forwarding mechanism. Greedy forwarding is the simplest mechanism, which sends packets to the closest neighbor node to destination. However, greedy forwarding does not guarantee destination reachability. Therefore, several forwarding mechanisms enhanced packet forwarding by involving new parameters and metrics (e.g. speed and direction) in the next forwarder selection process. However, with all the available enhancement of greedy forwarding, there is still a possibility for packet forwarding problems to occur but with lower probability. 


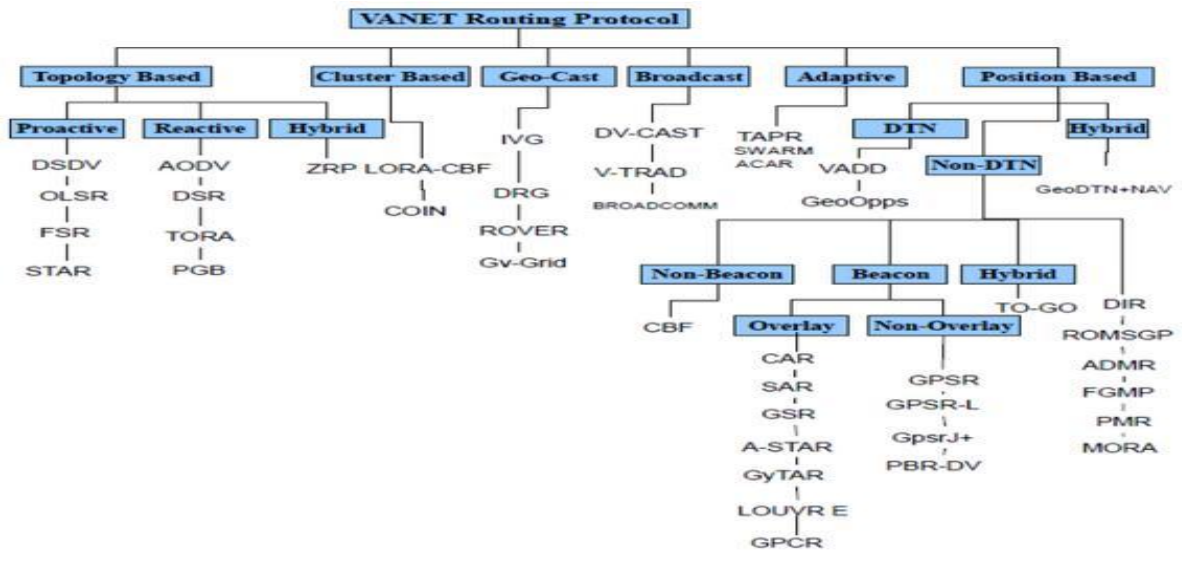

Fig. 2 Classification of Routing Protocols

\section{Classification of Routing Protocols}

For our convenience, routing protocols in VANET system are grouped into six categorized [5].

\section{Topology Based Routing}

It was developed to meet the dynamic nature of the Ad hoc networks. This routing protocol uses the topology which is existed in the network to forward the packets. Here periodic updating of routing protocols is required by constructing a table which contains routing paths of nodes. So consumption of lot of bandwidth for periodic updating of topology. Topology based routing protocols use links information to transmit the packets of data among nodes through the VANET. This TBR protocols are further divided as proactive and reactive routing protocols. Various types of routing protocols which are come under proactive category are DSDV, OLSR, FSR, CGSR, WRP and reactive routing protocols are AODV, TORA, PGB, and DSR. This category sub-divided into proactive, reactive, and hybrid routing sub-categories.

\section{Cluster Based Routing Protocols}

In this category, the network divides into clusters with similar characteristics like topology, speed, velocity etc. for intra cluster communication a virtual link is creased among nodes and for inter cluster communication a cluster communicates through cluster head. Examples of the Various Cluster based routing protocols are HCB, CBR, COIN, TIBCRPH, CBLR, CBDRP etc.

\section{Geocast Based Routing Protocols}

It is a location-based routing protocol. It delivers the packet from source to destination within a specified geographical area. The selected area for transmission is called ZOR (Zone of Relevance). Here, main idea is sender node need not to send packet to nodes beyond packet to overhead and network congestion when packets are flooded. Example of the various Geocast routing protocols are IVG, DGCASTOR, DRG etc.

\section{Position Based Routing Protocols}

It is a routing technique in which a node its routing decision with the help of GPS device. It sends packet from source to destination based on the geographic position of vehicle instead of using network address. It does not maintain either any link state information or routing table with another node in network. When the source needs to send a packet, it stores the destination address in the packet header.

\section{Broadcast Based Routing Protocols}

The simplest way to implement broadcasting is flooding. It is used by VANET for sharing information like its position, traffic, weather and emergency, road conditions among vehicles to maximum nodes possible. Flooding guarantees that the message will eventually reach all the nodes i.e. vehicles in the network. But in a large network, it causes exponential increase in message transmission resulting in collision so it increases the overhead and decreases the performance of network. Example for Broadcast routing protocols is BROADCOMM, UMB, EAEP, SRB, PBSM, PGB, DECA, POCA etc.

\section{Adaptive Connectivity Aware Routing (ACAR)}

The ACAR protocol combines two necessary elements by correctly selecting an optimal route, with the best estimated transmission quality and efficiency forwarding packets hopby-hop through each road segment in the selected route. To reduce the effects of imprecise statistical density data and development, the adaptive route selection algorithm gathers the real-time density information on-the-fly while forwarding packets.

The next hop is selected by using a metric that reduces the packet error rate (PER) of the whole route based on measured PERs at each node. It also handles the frequent network partition by carry-and-forward mechanism. ACAR is more suitable for suburban areas which do not require any infrastructure for the operation. It delivers with moderate control overhead and hence has a moderate delivery rate. The propagation mode for ACAR is unknown. 
These routing protocols depending on number of senders and receivers involved are divided into three types.

a. Unicast: This type of routing is used in $\mathrm{V} 2 \mathrm{~V}$ communication and is used to distribute the message to be specified area under in effective conditions like traffic jams, accidents etc. Here, the main goal is to perform data communication from a source node to a target node in the network via multi-hop wireless communication. The target node may be at either a precise known location or an approximate location within a specified range. Multicast is more suitable for application that require dissemination of messages to different nodes in VANET system [6].

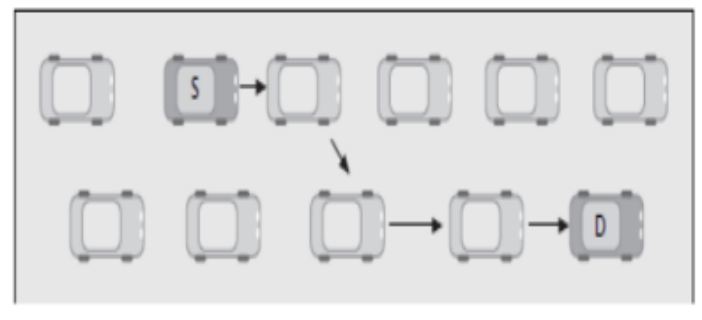

Fig. 3 Unicast Routing in VANET System

b. Multicast: It is a specialized form of multicast addressing in which a message sent to a group of target nodes in a geographic position, usually relative to the source of the message.

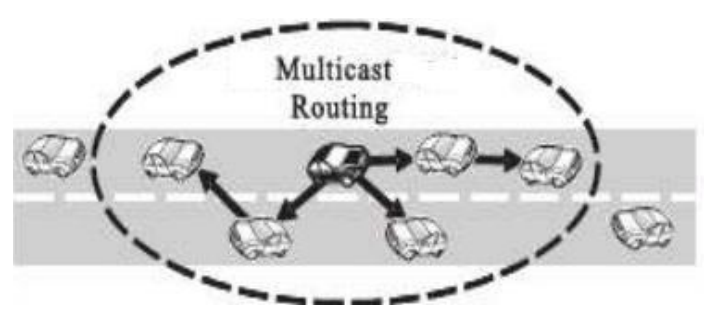

Fig. 4 Unicast Routing in VANET System

c. Broadcast/Geocast: The main feature is to have a source node sending information to all neighbors' nodes at once. The neighbor nodes that receive the broadcast message forward it through a new broadcast in order to deliver a message to the target nodes. Broadcast is used at the discovery phase of some routing protocols in unicast communication method to find an efficient route from the source vehicle to the target vehicle.

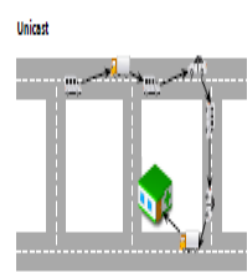

(a) Unicast

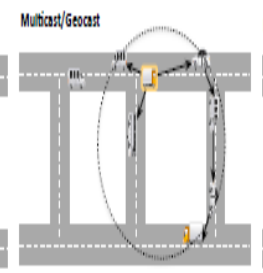

(b) Multicast/Geocast

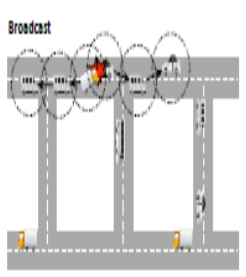

(c) Broadcast
Fig. 5 Different Communication Scenario in VANET System

The remainder of this paper is organized into as follows
Section II contains a review of related work. Section III explains routing protocols which are used in VANET communication. And we give conclusion in section IV, acknowledgment is given in section $\mathrm{V}$, and at last references are given which are used for preparing this paper.

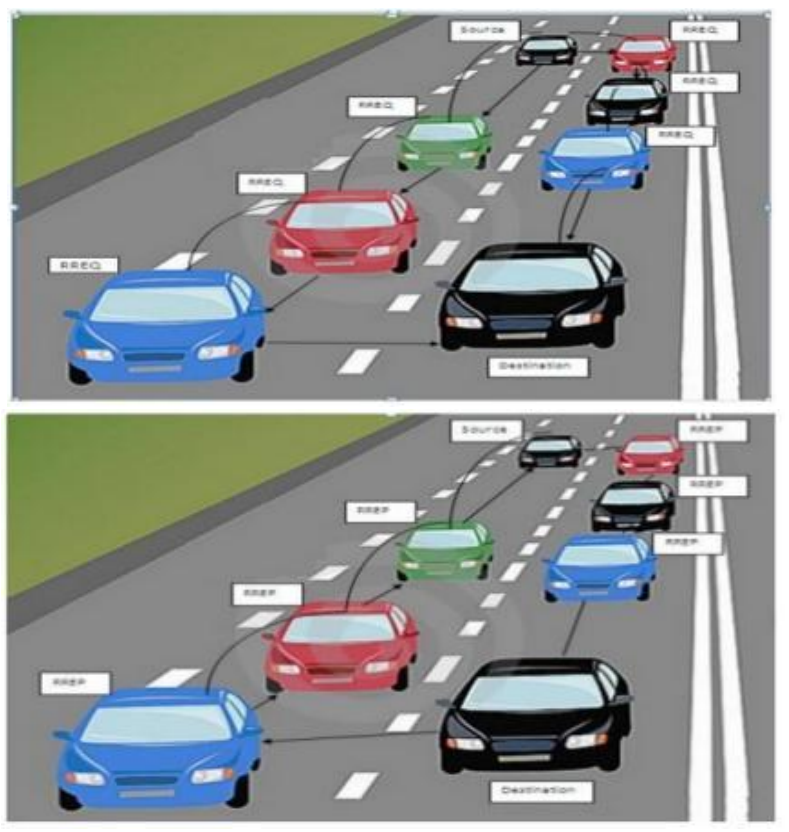

Fig. 7 Routing Propagation in VANET System

\section{RELATED WORK}

Data delivery in partially connected ad hoc networks is generally based on the store-and-forward concept. An Ad hoc network that uses the generalized message delay approach is called as DTMN (Delay Tolerant Mobile Network) [7]. In A-STAR, weights are assigned on the basis of traffic density and then. Here, using Dijkstra's algorithm, the shortest path is selected [8]. VADD is based on a carryand-forward scheme. This algorithm considers sparse network conditions but it was proposed for static destinations [9]. Shaffiee et al., proposed connectivity-aware minimum delay geographical routing using the same carryand-forward scheme to deliver packets from a moving vehicle to a fixed destination [10]. GeoDTN+Nav is an example of a hybrid protocol. It initiates a path when a node in VANET system transmits packet of data to another node [11]. Greedy perimeter coordinator routing (GPCR) is a position-based routing for urban environment [12]. GPSR protocol acts well in two scenarios, in highway and in city environments. GSR (Geographic Source routing) also uses Dijksta's algorithm to calculate shortest path consist of sequence intersection which packet has to traverse [13]. In recent years, various researches have examined various ad hoc routing protocols taking into consideration different performance metrics. Manvi et al., proposed a comparative study of three routing protocol: DSDV, AOMDV, and AODV [14]. Amit N. Chen Y. S analyzed the performance of AODV and DSR routing protocols using NS2 simulator with random waypoint model [15]. Goal.. A et al., proposed 
methods on how to propagate safety related messages in accidental areas [16]. Jerome Haerri et al. improved the performance of the AODV and OLSR routing protocols under two topical and realistic mobility models for VANET [17]. Nzouonta J. et al. proposed a road based vehicular traffic (RBVT) routing which is a class of VANET routing protocol for the city based environments [18]. Reza Fotohi et al. improved AODV routing protocol by Limiting Visited Hop Count mechanism [19]. Vidhale et al., evaluated the MANET routing protocols in VANET environment by using different mobility models available in VanetMobiSim [20].

\section{METHODS}

\section{A. General Classification of Routing Protocol}

In VANET technology typical routing protocols are classified as

TABLE I TYPICAL ROUTING PROTOCOLS

\begin{tabular}{|c|c|c|}
\hline Proactive routing & Reactive routing & Hybrid routing \\
\hline DSDV & AODV & GPSR \\
\hline OLSR & S-AODV & GPSR-L \\
\hline MOPR & PAODV & GPCR \\
\hline GSR & DSR & GPSRJ+ \\
\hline
\end{tabular}

\section{B. Proactive Routing (Table Driven)}

They maintain the global topology information in the form of tables at every time. Proactive routing protocols are mostly based on shortest path algorithm. Here, no route discovery is required. It requires low latency for real time applications. But problem in proactive routing is unused paths occupy a significant part of the available bandwidth.

1. DSDV (Destination Sequenced Distance Vector Routing): It is an enhanced form of Bellman-Ford algorithm where each node maintains a table which contains the shortest distance and the first node on the shortest path to every other node in the network. It can be applied to MANETs with few modifications.

2. OLSR (Optimized Link State Routing): It is a proactive protocol based on the table-driven methodology that floods a topology table of its neighbors to all nodes in the network. The link-state scheme is used by this protocol in an enhanced way to circulate topology information. OLSR operation fundamentally consists of servicing and updating information in a set of a table. This table are including data which is based on received control traffic, and control traffic is produced based on information returned from these tables. It increases the protocols suitability for ad hoc network with the rapid changes of the source and destination pairs. It uses three essential control message types
1. Topology Control message (TC): TC list out the nodes that had made the sender as their MPR.

2. HELLO Control message (HELLO): HELLO message will declare a node's knowledge about its surroundings.

3. Multiple interface Declaration messages (MID)

3. MPBR (Movement Prediction-Based Routing): It is based on vehicle movement information and guarantees the selection of the best next hop for data forwarding.

4. GSR (Global State Routing): This protocol for vehicular networks works very well in city environment where traffic density is high during day hours but less in night hours. GSR is combination of position based with topology based routing. This algorithm first selects the path and finds the shortest distance using Dijkstra algorithm. GSR uses Reactive Location Service (RLS) to get the destination address. This RLS provide best route for sender to send the packet. Application layer provide VANET applications to interact with user and other layers for communication. Transport layer uses protocols like User datagram protocol (UDP) and Transmission control protocol (TCP) for congestion control in VANET. Network layer provide route to the data by using RLS. The Last layer i.e. Physical layer provide wireless communication that is working on IEEE standard 802.11a, 802.11b, 802.11g.

\section{Reactive (On-Demand Routing Protocols)}

Here, the routes are established on demand and the destination sequence number can find the latest route to the destination.

1. AODV: Ad-hoc On Demand Distance Vector Routing protocol is depending on a mechanism related to on-demand approach. It is a reactive protocol which establishes a route when a node requires sending data packets. It has the ability of both multicast and unicast routing. It is different from others because it uses destination sequence number (DestSeqNum). It has up-to-date path to the destination because of using DestSeqNum. It responds to the link failure in the network. It can be applied to large scale Ad hoc network. It can be used in singular and multimode routing. It will create a (RREQ) route request message to be flooded to the other nodes in a limited way. It uses three message controls

1. Route request (RREQ)

2. Route reply (RREP)

3. Route error (RERR)

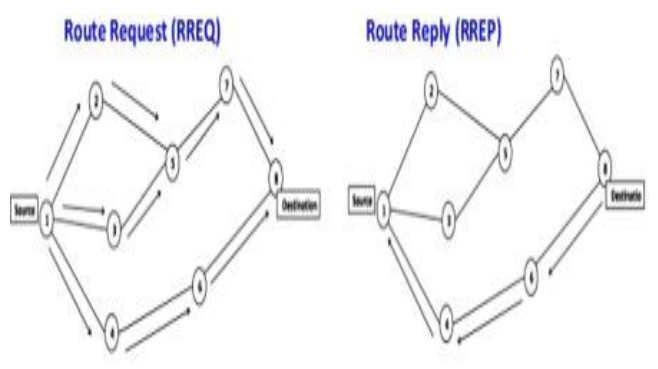

Fig. 8 AODV Routing Protocol Process 
If a node unable to send a packet, it generates a RERR message. When the originator node receives the RERR, it initiates a new route discovery for the given node.

\section{S-AODV (Secure AODV)}

The S-AODV routing protocol proposed in is used to protect the routing messages of the original AODV. We now explain the operation of the hash chains.

\section{3. $P-A O D V$}

Priority based route maintenance process uses overhearing mechanism in which the neighbor nodes overhear the data packets of a route. This mechanism is enabled by implementing the promiscuous mode in AODV routing protocol. The neighbor which overhears packets creates an ART (if not already created) and adds the entry for the node which it overheard, along with the destination information.

\section{DSR (Dynamic Source Routing)}

When a source node has data packets to be sent to that destination, it initiates route request packet. This route request flooded throughout the network. Each route request carries a sequence number on the packet before forwarding the packet. It utilizes source routing and maintains functional paths. It consists of route detection and route servicing. Here, a node requires four essential structures of data that are considered to be conceptual, to be able to engage in the DSR.

1. A Retransmission Buffer: The retransfer buffer of a node is packets queue sent by this node that is expecting for the arrival of an acknowledgement from the next hop in the source path.

2. A Send Buffer: Every packet after being registered in the buffer should be deleted from the send buffer.

3. A Route Cache: Route cache is responsible for storing all requested information related to routing in VANET system.

4. A Route Request Table: The route request table is considered of records collection about route request packets that were recently forwarded or originated by this node.

DSR contains two phases.

1. Route discovery

2. Route maintenance

\section{Hybrid Routing Protocol (HRP)}

It requires less memory and integrates both routing protocols advantages. It reports routing information only when there is a change in the topology of the network.

\section{GPSR (Greedy Perimeter Stateless Routing)}

When a packet reaches the region where greedy forwarding is impossible, this protocol is a restricted greedy forwarding procedure.

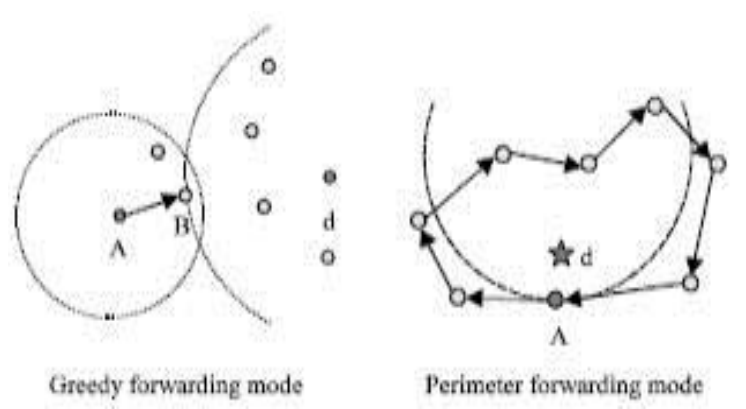

Fig. 9 GPSR modes

\section{OLSR (Optimized Link State Routing)}

It is a proactive protocol based on the table-driven methodology that floods a topology table of its neighbors to all nodes in the network. The link-state scheme is used by this protocol in an enhanced way to circulate topology information. OLSR operation fundamentally consists of servicing and updating information in a set of a table. This table is including data which is based on received control traffic, and control traffic is produced based on information returned from these tables. It increases the protocols suitability for ad hoc network with the rapid changes of the source and destination pairs. It uses three essential control message types

a. Topology Control message (TC): TC list out the nodes that had made the sender as their MPR.

b. HELLO Control message (HELLO): HELLO message will declare a node's knowledge about its surroundings.

c. Multiple interface Declaration messages (MID)

\section{GPCR}

The greedy perimeter coordinator routing (GPCR) protocol sends the data via the shortest path using the Dijkstra algorithm.

\section{GRP (Geographic Routing Protocol)}

It uses the concept of source routing and it uses GPS to locate the location of a node. In source routing the sender knows all hop-by-hop routes to the destination. All the routes are stored in the route cache. It has the capability to handle unidirectional links. This approach is known as hybrid routing protocol, because it uses simultaneously the strengths of reactive and proactive routing protocols. It is used in two into two approaches. In greedy forwarding, the data is sent to the closest neighbor of the destination node using the three VANET routing mechanisms. The second approach is perimeter routing which implies planar graph traversal concept, so any node does not require saving any extra or unimportant information. In order to use the greedy forwarding approach, the sender node determines the receiver node estimated location. The mostly used three greedy routing protocols are

1. Nearest with Forward Process (NFP)

2. Most Forwarded within R (MFR) and

3. Compass routing 
In perimeter approach, if the forward path cannot be determined by any node in the network. In GRP, two techniques are used are geographic forwarding, greedy forwarding.

\section{VADD (Vehicle-Assisted Data Delivery)}

This is an end-to-end connection in a network with tolerable delay. It chooses next hop based on the highest pre-defined direction priority.

\section{PDGR (Predictive Directional Greedy Routing)}

It is based on prediction but it is not reliable on all time and also it not gives the guarantee for delivery of packet to the node present in the transmission range of forwarding node.

\section{E. Cluster Based Routing protocols}

Besides these, clustering protocols role in VANET communication is also very important. Most used common type of clustering protocols is [21]

1. Cluster Base Routing Protocol (CBRP)

2. Clustering for Open IVC (Inter Vehicular Communication) Network (COIN)

3. LOcation Routing Algorithm with Cluster Based Flooding (LORA- CBF)

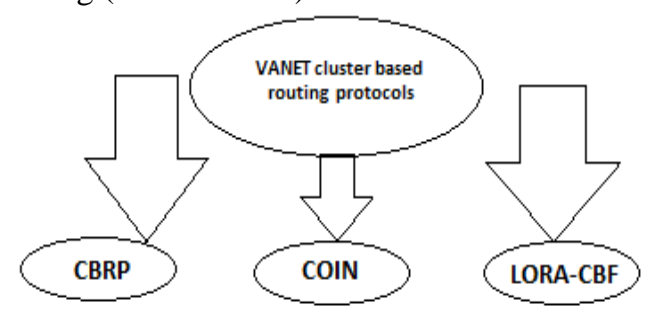

Fig. 10 VANET Cluster Based Routing Protocols

\section{CONCLUSIONS}

Routing is very important concept in VANET system. In achieving best communication among vehicles in VANET system, then routing plays very important role in VANET system. In this paper we discuss the main routing protocols anatomy (proactive routing, reactive routing, and hybrid routing) which are used in VANET system and anatomy of modern usable routing protocols.

\section{REFERENCES}

[1] Bijan Paul, Md. Ibrahim and Md. Abu NaserBikas, "VANET Routing Protocols: Pros and Cons", International Journal of Computer Applications (0975 -8887), Vol. 20, No. 3, April 2011.

[2] Cheng-Shiun Wu, Shuo-Cheng Hu andChih-Shun Hsu, "Design of Fast restoration multipath routing in VANETs", International Computer Symposium (ICS), IEEE,pp. 73-78, 2010.
[3] Bijan Paul and Mohammed J. Islam, "Survey over VANET Routing Protocol s for Vehicle to Vehicle Communication", IOSR Journal of Computer Engineering (IOSRJCE), Vol.7, No.5, pp. 01-09, 2012.

[4] Uma Nagaraj and PoonamDhamal, "Broudcasting routing protocols", 2011.

[5] W. Fan, Y. Shi, S. Chen and L. Zou, "A mobility metric based dynamic clustering algorithm (DCA) for VANETs", in Proceeding of the International Conference on Communication Technology and Application, Beijing, 2011, pp.752-756.

[6] Yuh-Shyan Chen, Yun-Wei Lin and Sing-Ling Lee,"A mobicast routing protocol with carry-and -forward in vehicular ad-hoc networks", $5^{\text {th }}$ International ICST Conference on Communications and Networking in China (CHINACOM), pp 1-5, 2010.

[7] K. Harras, K. Almeroth and E. Belding-Royer, "Delay Tolerant Mobile Networks (DTMNs): Controlled Flooding Schemes in Sparse Mobile Networks", IFIP Networking, 2005.

[8] Yuan Yang, MingweiXu, Dan Wang and Yu Wang, "Towards Energy-Efficient Routing in Satellite Networks", Selected Areas in Communications IEEE Journal, Vol. 34, No. 12, 20 Oct. - 1 Nov. 2016.

[9] J. Zhao and G. Cao, "VADD: Vehicle-Assisted Data Delivery in vehicular ad hoc networks", IEEE Transactions on Vehicular Technology, Vol. 57, No. 3, pp. 1910-1922, 2008.

[10] K. Shafiee and V. C. M. Leung, "Connectivity-aware minimum-delay geographic routing with vehicle tracking in VANETs", Ad Hoc Networks, Vol. 9, No. 2, pp. 131-141, 2011.

[11] B. Karp and H. T. Kung, "GPSR: Greedy Perimeter Stateless Routing for wireless networks", in Proceedings of the 6th Annual International Conference on Mobile Computing and Networking (MobiCom '00), Boston, Mass, USA, pp. 243-254, August 2000.

[12] S. A. Rao, M. Pai, M. Boussedjra and J. Mouzna, "GPSR-L: Greedy perimeter stateless routing with lifetime for VANETS", in Proceedings of the 8th International Conference on Intelligent Transport System Telecommunications (ITST '08), Phuket, Thailand, pp. 299-304, October 2008.

[13] E. H. Wu, P. K. Sahu and J. Sahoo, "Destination discovery oriented position based routing in VANET", inProceedings of the IEEE AsiaPacific Services Computing Conference (APSCC '08), Yilan, Taiwan, pp. 1606-1610, 2008

[14] S. Manvi, M. S. Kakkasageriand Mahapurush, "Performance analysis of AODV, DSR, routing protocols in vehicular Ad hoc network environment",International Conference on Future Computer and Communication, pp.21-26, 2009.

[15] Y. S. Chen, W. Lin and S. L. Lee, "A mobicast routing protocol for Vehicular Ad hoc Networks", ACM/Springer Mobile Networks and Applications, Vol.15, pp.20-35, 2010.

[16] A. Goal, K. G. Ramakrishnan, D. Kataria and D. Logothetis, "Efficient computation of delay-sensitive routes from one source to all destinations", In Proceedings of IEEE Conference on Computer Communications, pp.854-858, 2001.

[17] JoremeHaerri, "Performance Comparison of AODV and OLSR in VANETs Urban Environments under realistic mobility patterns", Department of Mobile Communications, pp.123-134, 2005.

[18] J. R. Nzouonta, N. Guiling and C. WangBorcea, "VANET Routing on city roads using Real-Time Vehicular Traffic information", in Proceeding of International Journal of Engineering Research and Technology, Vol. 2, No.3, pp.1-8, 2009.

[19] Reza Fatohi, ShaharamJamali, FatemeSarkohaki and ShabramBehzad, "An Improvement over AODV routing protocol by Limiting Visited hop count", in Proceedings of the I.J. Information Technology and Computer Science, Vol.9, pp.87-93, 2013.

[20] B. Vidhaleand S. S. Dorle, "Performance Analysis of routing protocols in realistic environment for Vehicular Ad hoc Networks, (VANET)", in Proceeding of International Conference on Systems engineering (ICSEngg.), Vol.2, pp.267-272, 2011.

[21] M. Rezaee and M. Yaghmaee, "Cluster based routing protocol for mobile ad hoc networks", INFOCOMP J. Comput. Sci. Vol. 8, pp. 30-36, 2009. 